\title{
Screening for alternative splicing of IncRNA Dleu 2 in the mouse liver cell line AML-12
}

\author{
MATTHEW KRIVACKA KAY, JIAN ZHANG and MAHUA CHOUDHURY \\ Irma Lerma Rangel College of Pharmacy, Texas A\&M University, College Station, Texas, TX 77843, USA
}

Received November 25, 2020; Accepted March 19, 2021

DOI: $10.3892 /$ br.2021.1426

\begin{abstract}
The long non coding RNA deleted in leukemia 2 gene (Dleu2) has recently been demonstrated to be an active player in the progression of several types of cancer, including hepatocellular carcinoma. Dleu2 may serve a role in modulating the downstream effects-mediated by alternative splicing of its multiple exons. However, the proportional expression of these alternative splicing populations of the Dleu2 exons is currently unknown. To determine how Dleu2 could be affected by alternative splicing, a series of alternative splicing primer sets were designed to investigate which transcripts were preferentially activated when Dleu2 was targeted for downregulation or upregulation. A specific Dleu2 small interfering RNA that targeted an exon upstream of the tumor suppressor microRNA site significantly knocked down Dleu2 expression across all the primer sets used, which targeted 13 different alternative splicing transcripts over 5 different promoter sites in the mouse liver cell line, AML-12. Similarly, $50 \mu \mathrm{M}$ Resveratrol led to significant upregulation of Dleu2 in 11 alternative splicing transcripts. These results show that Dleu2 is capable of successful modulation across alternative splicing transcripts that can be screened, and also that Resveratrol can be a potential nutraceutical, which may potentially lead to novel approaches in the use of lncRNA Dleu2 for diagnostics and regulation.
\end{abstract}

\section{Introduction}

Deleted in leukemia 2 gene (DLEU2) is a long noncoding RNA (lncRNA), which has been shown to serve a role as a tumor suppressor gene in several types of blood cancer $(1,2)$. Chronic lymphocytic leukemia is characterized by DLEU2 deletion

Correspondence to: Dr Mahua Choudhury, Irma Lerma Rangel College of Pharmacy, Texas A\&M University, 206 Olsen Boulevard, 336 Reynolds Medical Sciences Building, College Station, Texas, TX 77843, USA

E-mail: mchoudhury@tamu.edu

Key words: long non-coding RNA deleted in leukemia 2 gene, alternative splicing, Resveratrol as well as its tumor suppressor microRNAs (miRNAs/miRs) miR-15a/16-1 region in $\sim 55 \%$ of cases $(3,4)$. As a tumor suppressor gene, the DLEU2/miR-15a/miR-16-1 locus has been extensively characterized (5-7). Since the DLEU2/miR15a/16-1 locus is present in several somatic tissues, it also has shown to serve a role in regulating cell proliferation in various cell types including, vascular endothelial cells (8), various lymphomas and leukemias (9), as well as hepatocytes (10). Physiologically, miR-15a and miR-16-1 are upregulated as a miRNA cluster from the intronic 13q14.3 region of the DLEU2 lncRNA (10). DLEU2 itself has also been implicated in the sponging of several miRNAs, including miR-30a (11) and miR-455, which can regulate several downstream functions (12). However, to the best of our knowledge, there are no studies that have assessed the effects of lncRNA DLEU2 alternative splicing in the liver.

Large-scale sequencing projects over the last decade have demonstrated the extent of alternative splicing of mammalian transcripts. Studies have shown that $>95 \%$ of human genes generate transcripts that are alternatively spliced (13-15). However, not all alternatively spliced transcripts produce functional proteins (16-18). In humans, numerous studies have demonstrated an association between dysregulation of RNA splicing and the development/progression of several diseases $(19,20)$. Certain lncRNAs have been shown to serve a crucial role in the regulation of alternative splicing in response to several stimuli or during disease (21-23). Interestingly, Dleu2 lncRNA in mice is differentially regulated, with 15 different transcripts available over five different promoter sites (Fig. S1; from ENSEMBL database: uswest.ensembl. org/Mus_musculus/Gene/Summary?db=core;g=ENSMUSG 00000097589;r=14:61602839-61682373) (24). MiR-15a/15b and miR-16 are encoded by introns downstream of exons present on some, but not all, of the Dleu2 transcripts (denoted by a yellow star in Fig. S1). It has previously been shown that the DLEU2/miR-15a/miR-16-1 locus contributed to cell apoptosis and progression in liver fibrosis (10), modulated miR-30a sponging in clear cell renal cell carcinoma (11), and miR-15a/16 suppression was implicated in the development of pleural mesothelioma (25).

Dependent on the size of the introns spliced out, and the exons that are retained, Dleu2 alternative splicing can have different functions on lncRNA/RNA/miRNA regulation inside the cell. In an in silico study by Ma et al (26), RNA sequencing (RNASeq) platforms showed that exon 9 of DLEU2 was a better marker than total DLEU2 expression for 
predicting unfavorable overall survival rates in patients with esophageal adenocarcinoma. Though methylation analysis of the DNA region encoding Dleu2 lncRNA with regard to several cancer types, including esophageal adenocarcinoma (26), and pediatric acute myeloid leukemia (27) have been performed, no specific studies assessing the alternative splicing populations of Dleu2 in the liver or any other tissues were found. In the present study, it was shown that Dleu 2 alternative splicing transcripts were affected by silencing or overexpression, and this may improve our understanding of how Dleu2 dysregulation and modulation affect progression of various diseases.

\section{Materials and methods}

Experimental sample used for comparison of Dleu2 splicing primer sets. In vitro experiments were performed on the mouse liver cell line AML-12 using small interfering (si) RNA knockdown for downregulation of Dleu2 expression, and upregulation was achieved by treating cells with trans Resveratrol antioxidant.

Cell lines and reagents. AML-12 (ATCC ${ }^{\circledR}$ CRL-2254), the $\alpha$-mouse liver cell line 12 , was cultured in complete media (DMEM/F12 supplemented with $10 \%$ FBS, $1 \%$ penicillin/streptomycin, 1X Insulin/Transferrin/Selenium solution and $40 \mathrm{ng} / \mathrm{ml}$ Dexamethasone) as recommended by the supplier (all form ATCC). SiRNA transfections were performed using OptiMEM media, Lipofectamine ${ }^{\circledR}$ RNAiMAX, Silencer Select Negative Control \#1 (cat. no. 43908435 nm) and mouse Silencer Select n410472 (cat. no. \#4390771; sense, UGCUCUUAAUAAGCAUUAAtt; antisense, UUAAUGCUUAUUAAGAGCAgc; $5 \mathrm{~nm}$ ) (all from Thermo Fisher Scientific, Inc. Trans Resveratrol (cat. no. R5010-100MG) was obtained from Sigma-Aldrich; Merck KGaA.

siRNA transfections. AML-12 cells were seeded at a density of $5 \times 10^{5}$ cells/well in a 12 well plate and allowed to grow to $70-80 \%$ confluency overnight at $37^{\circ} \mathrm{C}$ in complete media. After $24 \mathrm{~h}$, the media was removed and cells were washed $3 \mathrm{x}$ with room temperature (RT) 1X PBS, after which pre-warmed $\left(37^{\circ} \mathrm{C}\right)$ OptiMEM was added. Dleu 2 siRNA or Negative Control \#1 was added to each well to a final concentration of $25 \mathrm{nM}$ per $\mathrm{ml}$ according to manufacturer's protocol (Thermo Fisher Scientific, Inc.). After incubation at $37^{\circ} \mathrm{C}$ in $5 \% \mathrm{CO}_{2}$, RNA was extracted from cells after $24 \mathrm{~h}$ of transfection.

Trans Resveratrol exposure. In an unrelated project, the effects of several antioxidants on Dleu2 were investigated. Interestingly, it was identified that Resveratrol, a potent anti-oxidant, led to an increase in Dleu2 expression. This upregulation of DLEU2 by Resveratrol was also observed in the human hepatocellular carcinoma cell line HepG2 (data not shown). Therefore, Resveratrol was used as a positive technical control in the present study.

AML-12 cells were seeded as described above. A total of $1 \mathrm{ml}$ complete media containing $0.1 \% \mathrm{EtOH}$, or $50 \mu \mathrm{M}$ trans Resveratrol in $\mathrm{EtOH}$ was added to each well, and incubated for $20 \mathrm{~h}$ at $37^{\circ} \mathrm{C}$.

RNA extraction and reverse transcription-quantitative (RT) $q-P C R$. RNA was extracted using the Qiagen RNeasy
Mini Prep kit (Qiagen, GmbH), according to the manufacturer's protocols, and RT-qPCR was performed as previously described, using the primer sequences designed for alternative splicing and the other sequences shown below (28).

Alternative splicing primer design and primer sets. SiRNAs were developed to specifically target the mouse Dleu2 exon (Mouse chromosome 14, Exon 4, 61,632,437-61,632,483 bp) directly upstream of the miR-15a intron site (Thermo Fisher Scientific, Inc.). Alt1 (Dleu2 alias) primers for mouse (m) mDleu2 were designed using NCBI BLAST, targeting the largest cDNA sequences in their database (National Institutes of Health). Alt1 was designed to bind to 4 of the Dleu2 transcripts at the same time in ENSEMBL; however, this primer set did not target several of the other alternative Dleu2 splicing sets in the mouse ENSEMBL database (ensembl.org). Therefore, 15 different spliced Dleu 2 sequences were investigated, with spliced exon-exon cDNA sequences retrieved from the ENSEMBL website, and specific primer sets designed to target different Dleu2 transcripts.

These cDNA sequences were sequentially run on NCBI BLAST to assess the similarity, with only outlier exon regions not matching any other mDleu 2 cDNA sequences selected. However, several primer sets still had matching regions on the larger -202 and -207 cDNA sequences yet still targeted their main splicing sequence; these primer sets were kept. These available selected areas were then screened using Primer3Plus (primer3plus.com) to find regions capable of producing standard PCR products (20-25 bp primer length, $\mathrm{T}_{\mathrm{m}} 60^{\circ} \mathrm{C}, 60 \% \mathrm{GC}$ content, 50-200 bp product size). These targeted primer sets were then screened against the BLAST alignment tool in ENSEMBL to check for other possible DNA matches in the mouse genome. Primer sets with low E values (below 0.05) that were specific for the mDleu2 gene were kept while others were discarded. Primers that matched all these criteria (Table SI) were developed and purchased from Sigma-Aldrich; Merck KGaA for use in the qPCR. Ineffective primer pairs that resulted in multiple melt curve peaks during qPCR amplification were discarded post-testing. Primers targeting specific Dleu2 transcripts are shown outlined in various colors on the ENSEMBL in Fig. 1B.

Expression of genes associated with proliferation. RT-qPCR was also used to measure expression of genes associated with proliferation, including proliferating cell nuclear antigen, transforming growth factor- $\beta$ and epidermal growth factor receptor (EGFR) in Dleu2-silenced and control AML-12 cells. The sequences of the primers used are listed in Table SI.

Statistical analysis. All data are presented as the mean \pm the standard error of the mean. Comparisons between groups were performed using a Student's t-test (two groups). $\mathrm{P}<0.05$ was considered to indicate a statistically significant difference. Statistical analysis was performed using GraphPad Prism version 6.0 (GraphPad Software, Inc.).

\section{Results}

Alternative splicing modulates Dleu2 for targeted primers. The Alt1 primer set targets mDleu2-208, -203, -210 and -211, 
A a

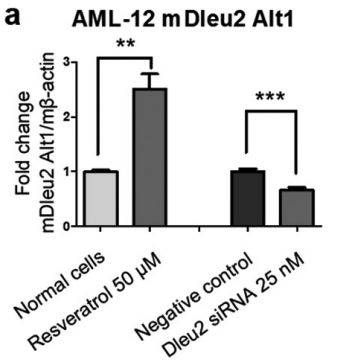

e AML-12 mDleu2-201 119 bp

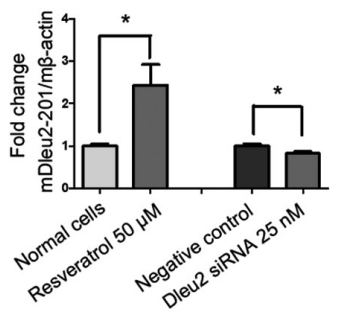

I AML-12 mDleu2-202+207 137 bp

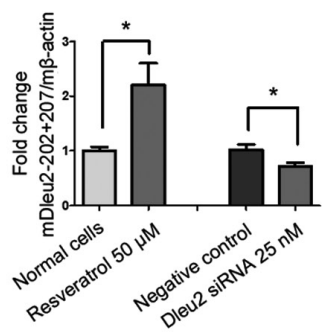

b AML-12 mDleu2-208 117 bp

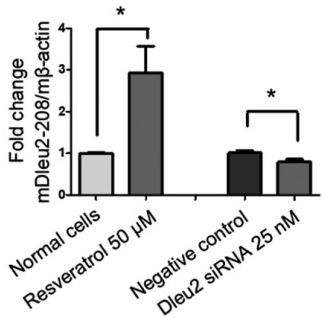

f AML-12 mDleu2-204 113 bp
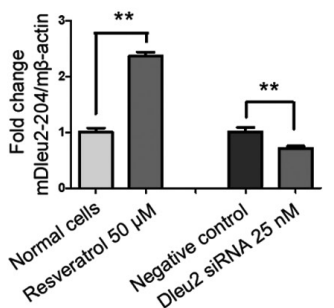

j AML-12 mDleu2-209 71 bp

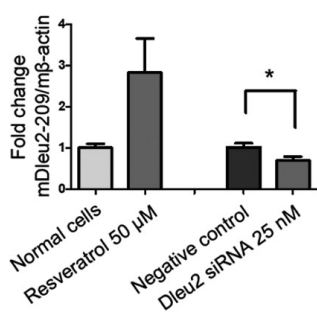

C AML-12 mDleu2-203 51 bp
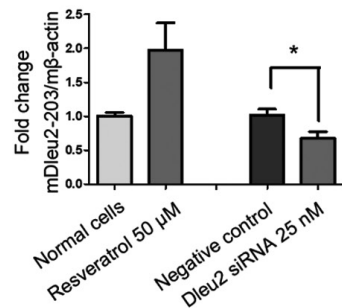

g AML-12 mDleu2-215 TEC 145 bp

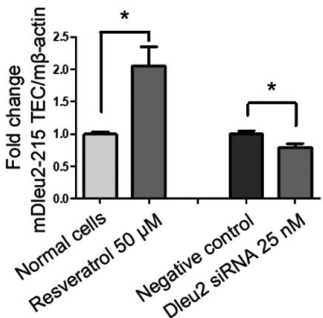

K AML-12 m-Dleu2-Gm27010-201 101 bp

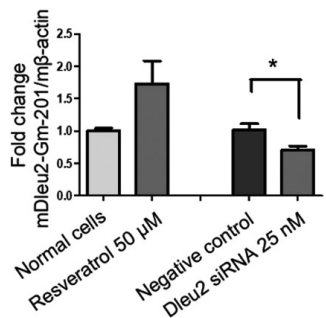

d AML-12 m-Dleu2-210 118 bp

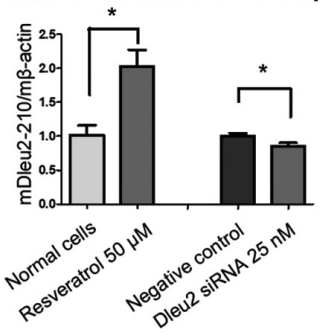

h AML-12 mDleu2-213 149 bp

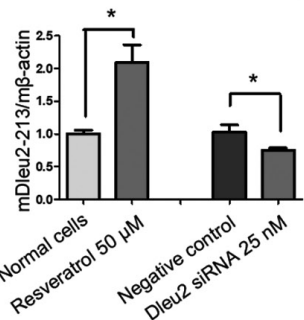

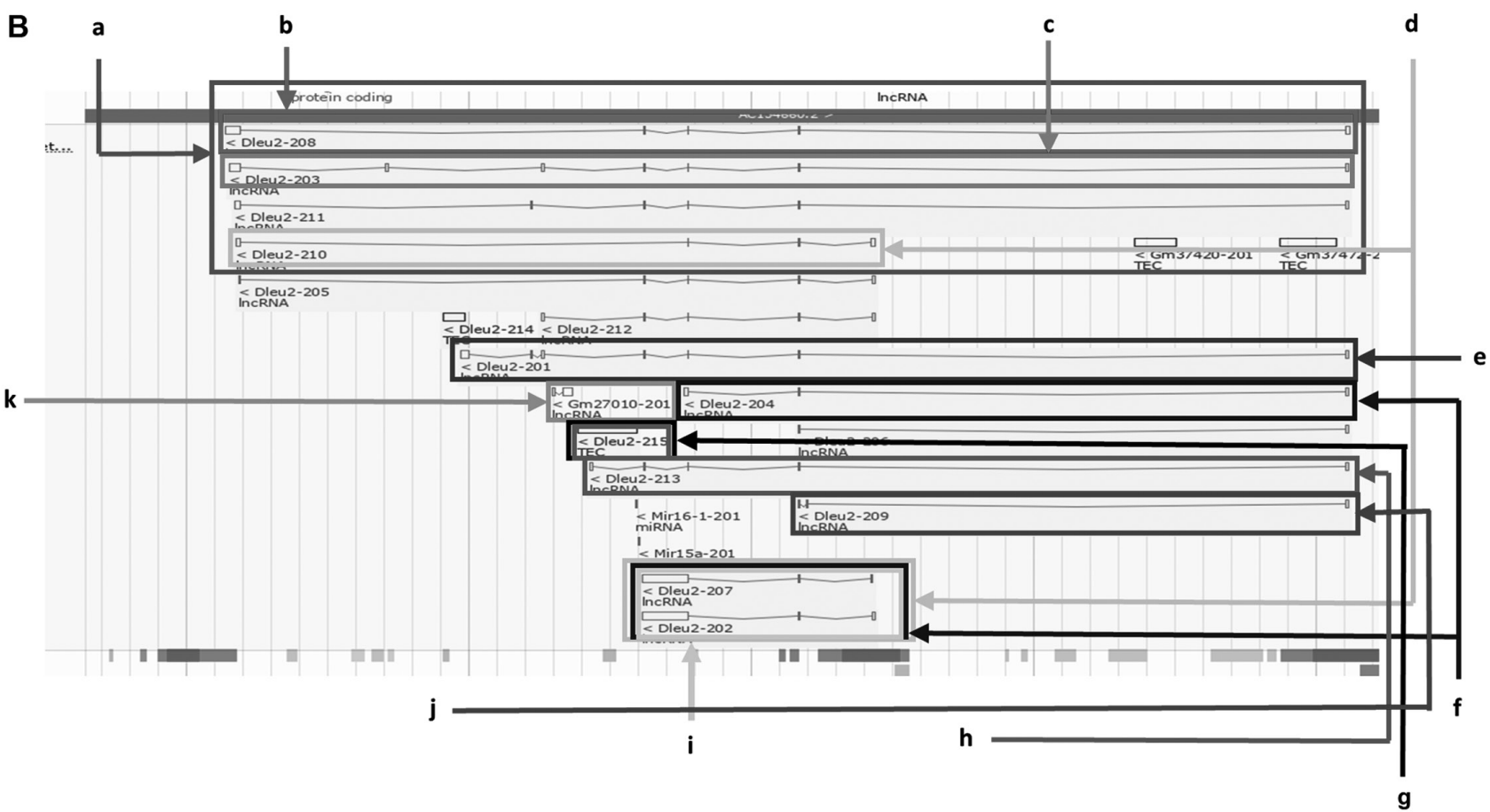

Figure 1. AML-12 mDleu2 alternative splicing primer set results. (A) In vitro AML-12 mDleu2 expression assessed using the different primer sets in cells treated with $50 \mu \mathrm{M}$ Resveratrol compared with control cells ( $\mathrm{n}=3$, left), and in cells transfected with Dleu2 siRNA compared with the negative control $(\mathrm{n}=6$, right). (B) Targeted primer binding sites are shown on ENSEMBL mDleu2 alternative splicing site map: (a) mDleu2-Alt1 primer set; (b) mDleu2-208 primer set; (c) mDleu2-203 primer set; (d) mDleu2-210 primer set; (e) mDleu2-201 primer set; (f) mDleu2-204 primer set; (g) mDleu2-215 TEC primer set; (h) mDleu2-213 primer set; (i) mDleu2 202+207 primer set; (j) mDleu2-209 primer set; (k) mDleu2 Gm27010-201 primer set. Data presented as the mean \pm the standard error of the mean of at least 3-6 independent experiments. ${ }^{*} \mathrm{P}<0.05,{ }^{* * *} \mathrm{P}<0.01,{ }^{* * * *} \mathrm{P}<0.001$. The splicing maps in panel (B) were obtained from ENSEMBL (uswest.ensembl.org/Mus_musculus/Gene/Summary?db=core;g=ENSMUSG00000097589;r=14:61602839-61682373). siRNA, small interfering RNA; Dleu2, deleted in leukemia 2 gene.

four of the mDleu 2 transcripts produced in the Dleu2 transcript map with overlapping regions (Fig. 1Ba). As such, this primer set provides a general outlook on how mDleu2 is expressed overall in the mouse under downregulating/upregulating 
experimental conditions. Resveratrol treatment for $20 \mathrm{~h}$ resulted in significant upregulation $(\mathrm{P}<0.01)$ in the mDleu2 Alt1 subset, and transfection of mDleu2 siRNA significantly downregulated its expression $(\mathrm{P}<0.001$; Fig. 1Aa). The mDleu2-208 primer set only targets mDleu2-208 in the Dleu2 transcript map (Fig. 1Ab and $\mathrm{Bb}$ ). After $20 \mathrm{~h}$ Resveratrol treatment significant upregulation $(\mathrm{P}<0.05)$ in the mDleu2-208 subset was observed, whereas siRNA-mediated knockdown resulted in significant downregulation $(\mathrm{P}<0.05)$, although the effect was less potent than that on the Alt1 set. The mDleu2-203 primer set only targets mDleu2-203 in the Dleu2 transcript map (Fig. 1Ac and Bc). The Resveratrol treatment had no significant effect on the mDleu2-203 subset, whereas mDleu2 siRNA resulted in knockdown $(\mathrm{P}<0.05)$, although the effect was less potent than that on the Alt1 set. The mDleu2-210 primer set targets mDleu2-210, and overlaps -202 and -207 in the Dleu2 transcript map (Fig. 1Ad and 1Bd). Resveratrol treatment resulted in significant upregulation $(\mathrm{P}<0.05)$ in the mDleu2-210 subset, and mDleu2 siRNA knockdown resulted in significant downregulation $(\mathrm{P}<0.05)$, although it was less significantly associated than that of the Alt1 set.

The mDleu2-201 primer set only targets mDleu2-201 in the Dleu2 transcript map (Fig. 1Ae and Be). Resveratrol treatment resulted in significant upregulation $(\mathrm{P}<0.05)$ in the mDleu2-201 subset, and mDleu2 siRNA knockdown resulted in significant downregulation $(\mathrm{P}<0.05)$. The mDleu2-204 primer set targets mDleu2-204, and overlaps part of -202 and -207 in the Dleu2 transcript map (Fig. 1Af and Bf). Resveratrol exposure resulted in significant upregulation $(\mathrm{P}<0.01)$ of the mDleu2-204 subset, and mDleu2 siRNA knockdown significantly downregulated expression of this subset $(\mathrm{P}<0.01)$.

The mDleu2-215 TEC primer set, mDleu2-213 primer set, and mDleu2-202+207 primer set targets mDleu2-215 TEC, mDleu2-213 and overlaps -215 TEC, and mDleu2-202 and -207 in the Dleu2 transcript map, respectively (Fig. $1 \mathrm{Ag}$ and $\mathrm{Bg}$; Fig. 1 Ah and Bh; Fig. 1Ai and Bi). Resveratrol treatment resulted in significant upregulation $(\mathrm{P}<0.05)$ of the mDleu2-215 TEC subset, mDleu2-213 subset, and mDleu2-202+207 subset; and mDleu2 siRNA knockdown resulted in significant downregulation of all three sets $(\mathrm{P}<0.05)$.

The mDleu2-209 primer set targets mDleu2-209 in the Dleu2 transcript map (Fig. 1Aj and Bj). Resveratrol exposure did not significantly affect the mDleu2-209 subset; however, the mDleu2 siRNA did significantly downregulate its expression $(\mathrm{P}<0.05)$. The mDleu2-Gm27010-201 primer set only targets mDleu2-Gm27010-201 in the Dleu2 transcript map (Fig. 1Ak and Bk). Resveratrol treatment did not have a significant effect on the mDleu2-201 subset, whereas mDleu2 siRNA did significantly downregulate its expression $(\mathrm{P}<0.05)$.

Expression of genes associated with proliferation. Expression of proliferating cell nuclear antigen, transforming growth factor- $\beta$ and epidermal growth factor receptor was not altered significantly when Dleu2 expression was knocked down (Fig. S2).

\section{Discussion}

DLEU2 is a lncRNA that was only discovered a decade ago, and has more recently been implicated in several types of cancer, including multiple types of blood cancer, as well as hepatocellular carcinoma. As such, it is possible that changes to the expression patterns of one or several of its multiple transcripts, and thus dysregulation of the downstream lncRNAs, miRNAs and/or RNAs interactions, may lead to abnormalities. Dleu 2 has been shown to regulate several physiological pathways, including proliferation (29-32), although, it does not alter proliferation of the non-cancerous AML-12 cells (Fig. S2). Interestingly, Dleu2 has a unique alternative splicing transcriptome, with multiple patterns for exon-exon splicing and intron removal $(2,26)$. How these populations of various transcriptions arise may be important in interpreting the pathological outcomes. For example, changes in the expression levels of various exons within Dleu2 have been shown to be correlated with unfavorable overall survival rates in patients with esophageal adenocarcinoma (26). Therefore, the ability to monitor and investigate changes to these expression patterns or alternative transcription motifs may be of use in identifying dysregulated cellular biology and cancer progression.

Based on the results of the individual alternative splicing primer sets, a repeating pattern for all lncRNA Dleu 2 primers used to measure Dleu 2 transcript expression was observed. The mDleu2-Alt1 primer set targeted the mDleu2-208, -203, -210 and -211 transcripts, and the expression of these transcripts increased when treated with Resveratrol, and decreased when expression was knocked down using siRNAs. Most individual primer sets displayed this same pattern with significant upregulation of mDleu2 upon exposure to Resveratrol, and significant downregulation in the siRNA knockdown experiments, although there were some exceptions; specifically Resveratrol did not result in significant downregulation of mDleu2-203, -209 and -Gm27010-201, a downward trend was observed in these cases as well. Whereas the Resveratrol results showed some inconsistencies, what was noteworthy about these results was that in the Dleu2 transcripts which lacked the Exon 4 sequence, the target of the siRNAs, they were still knocked down significantly overall. Specifically, mDleu2-210,-204,-202+207, -209 and -Gm27010-201 transcripts lacked Exon 4 from the ENSEMBL alternative transcription map (Fig. S1), yet were still significantly knocked in the cells transfected with the siRNAs. It is possible that there are interactions present between the various alternative transcripts that modulate the expression of the entire Dleu2 transcript family, or perhaps the targeted siRNAs also targeted other areas similar to Exon 4 on the other transcripts. Future experiments using other targeted siRNAs that specifically target other exons on Dleu2 may provide additional information as to how this principle within Dleu2 is regulated.

Overall, the results found evidence of Dleu2 modulation across the spectrum of transcripts, with upregulation due to Resveratrol exposure and downregulation due to siRNA treatment. Upregulation of Dleu2 occurred across all transcripts generally uniformly (though not always significantly), with increased lncRNA expression from the 5'-promoter sites available (Fig. S1, bottom panel, red bars). c-Myb and PPAR are known to be positive regulators of these promoter binding sites in Dleu2 (33). Furthermore, noted shifts in splice patterns are present in diseased states as compared with the global splicing map of the human genome, which can modulate the frequency of Poly-A choices on transcripts (21). 
It is possible that the limited changes in Dleu2 expression from $20 \mathrm{~h}$ treatment with Resveratrol and $24 \mathrm{~h}$ siRNA silencing treatments using the individual transcript primer sets may be due to the limited time span of the AML-12 cell culture experiments, and that longer exposures may have resulted in more significant results. Some transcripts, particularly mDleu2-203, -209 and -Gm27010-201, were not as significantly affected by Resveratrol treatment relative to the others, perhaps indicating more resistance to changes in expression compared with the other transcripts. As specific changes to DLEU2 exon expression have been confirmed in esophageal adenocarcinoma (26) and pediatric acute myeloid leukemia (27), exon expression changes are definitely a possibility in altering liver Dleu2 transcript expression. However, more specific testing is required to determine these alternative splicing changes in future experiments, perhaps using RNASeq to sidestep the limitations of overlapping targeted primer sets on the nested mouse Dleu2 transcripts. Additionally, several of these initial results need be assessed and confirmed in vivo or even using human liver tissues to identify the effects of alternative splicing.

A previous study looked at a novel alternative splicing subset within an overlapping DLEU2/LEU5/RFP2 cluster, containing multiple alternative splicing sites to produce monocistronic transcripts or a bicistronic transcript (34). Primers were designed to individually target exons of the LEU5/RFP2 cluster as well of that of DLEU2 and the overlapping sequences between the two genes. Whilst this previous study involved artificial constructs of areas surrounding exons from DLEU2/LEU5/RFP2, they were able to confirm specific intronic sequences between exons using RT-qPCR. The present study only investigated Dleu2 sequences within specific unique exons, whereas the previous study was searching for sequences spanning multiple exons, although RT-qPCR was used in both studies to build a larger exon-exon map of alternative splicing regions within and surrounding lncRNA Dleu2.

In other studies involving pediatric AML (27) and esophageal adenocarcinoma (26), alternative splicing of DLEU2 was investigated, and hypermethylation or expression of specific exons was shown to be associated with cancer progression. As such, there may be numerous other mechanisms available, in addition to simple alternative splicing, by which Dleu2 promotes the development and/or progression of cancer.

The way in which both mouse and human Dleu2 and DLEU2, respectively, are organized in their promoter regions and location/number of alternative transcription sites may exert an effect on how DLEU2 is modulated between the two species (24). Mouse Dleu2 is primarily layered such that most of the functional transcripts reside nested within one another, thus it is difficult to find specific primer sets that will only target one transcript and does not overlap multiple transcripts. As such, there are more promoter sites, and a more spread out area for human DLEU2 alternative splicing sites (Fig. S3A and B). Whereas the miR-15a/16 locus is also present for a simple majority of the human DLEU2 alternative splicing sites, there are multiple DLEU2 alternative splicing sites coded outside of that region in humans (24). It is probable that with the additional human DLEU2 transcripts compared to mouse Dleu2 regulation, there may be additional modulatory factors present in the regulation of human Dleu2, particularly through different alternative splicing transcription patterns, or through other dysfunctional regulatory factors. Finding these transcriptional changes within transcriptome populations could ultimately lead to novel diagnostic tools and aid in prevention of disease.

In conclusion, using the splicing map of Dleu2, the present study was the first to show the modulation of Dleu2 alternative spliced region expression was shown through knockdown and overexpression. Furthermore, a nutraceutical, Resveratrol, was shown to increase the expression of Dleu2. This may assist in identifying a means of cancer prevention for certain types of cancer. Recently, Soreq et al (22) used wide annotations of alternate promoters, splicing and alternative poly-A sites to identify and quantify both disease- and treatment-induced splicing shifts, miRNA binding site modifications, putatively changed protein-protein interactions and other transcript structural changes in Parkinson's leukocytes. Future studies in in vivo mouse and human samples should expand in the above directions to investigate Dleu2 and alternative splicing in depth.

\section{Acknowledgements}

We would like to thank the undergraduate lab assistants Miss Sumiya Wahab, Miss Jazmin Diaz, Miss Victoria Tilson and Miss Faith Upton (Texas A\&M University) for their contributions to this work.

\section{Funding}

The present study was supported by funding from the Morris L Lichtenstein Jr. Medical Research Foundation (grant no. M1500307).

\section{Availability of data and materials}

The datasets used and/or analyzed during the present study are available from the corresponding author on reasonable request.

\section{Authors' contributions}

MC and MKK designed the study and wrote the manuscript. MKK performed the experiments. JZ analyzed and interpreted the data. All authors read and approved the final manuscript. MKK, JZ and MC confirmed the authenticity of all the raw data.

\section{Ethics approval and consent to participate}

Not applicable

\section{Patient consent for publication}

Not applicable.

\section{Competing interests}

The authors declare that they have no competing interests.

\section{References}

1. Klein UL, Lia M, Shen Q, Smith PM, Tang H, Mo T, Crespo M, Siegel R, Bhagat G and Dalla-Favera R: The DLEU2/Mir-15a/ Mir-16-1 locus, commonly deleted in B-cell chronic Lymphocytic leukemia (CLL), controls B-cell compartment expansion and its deletion leads to CLL in mice. Blood 112: 25, 2008. 
2. Klein U, Lia M, Crespo M, Siegel R, Shen Q, Mo T, Ambesi-Impiombato A, Califano A, Migliazza A, Bhagat G, et al: The DLEU2/miR-15a/16-1 cluster controls B cell proliferation and its deletion leads to chronic lymphocytic leukemia. Cancer Cell 17: 28-40, 2010.

3. Döhner H, Stilgenbauer S, Benner A, Leupolt E, Kröber A, Bullinger L, Döhner K, Bentz M and Lichter P: Genomic aberrations and survival in chronic lymphocytic leukemia. N Engl J Med 343: 1910-1916, 2000.

4. Kalachikov S, Migliazza A, Cayanis E, Fracchiolla NS, Bonaldo MF, Lawton L, Jelenc P, Ye X, Qu X, Chien M, et al: Cloning and gene mapping of the chromosome 13q14 region deleted in chronic lymphocytic leukemia. Genomics 42: 369-377, 1997.

5. Bullrich F, Fujii H, Calin G, Mabuchi H, Negrini M, Pekarsky Y, Rassenti L, Alder H, Reed JC, Keating MJ, et al: Characterization of the 13q14 tumor suppressor locus in CLL: Identification of ALT1, an alternative splice variant of the LEU2 gene. Cancer Res 61: 6640-6648, 2001

6. Corcoran MM, Rasool O, Liu Y, Iyengar A, Grander D, Ibbotson RE, Merup M, Wu X, Brodyansky V, Gardiner AC, et al: Detailed molecular delineation of 13q14.3 loss in B-cell chronic lymphocytic leukemia. Blood 91: 1382-1390, 1998.

7. Liu Y, Corcoran M, Rasool O, Ivanova G, Ibbotson R, Grandér D, Iyengar A, Baranova A, Kashuba V, Merup M, et al: Cloning of two candidate tumor suppressor genes within a $10 \mathrm{~kb}$ region on chromosome $13 \mathrm{q} 14$, frequently deleted in chronic lymphocytic leukemia. Oncogene 15: 2463-2473, 1997.

8. Li Y, Sun N, Lu Z, Sun S, Huang J, Chen Z and He J: Prognostic alternative mRNA splicing signature in non-small cell lung cancer. Cancer Lett 393: 40-51, 2017.

9. Lerner M, Harada M, Lovén J, Castro J, Davis Z, Oscier D, Henriksson M, Sangfelt O, Grandér D and Corcoran MM: DLEU2, frequently deleted in malignancy, functions as a critical host gene of the cell cycle inhibitory microRNAs miR-15a and miR-16-1. Exp Cell Res 315: 2941-2952, 2009.

10. Jiang XP, Ai WB, Wan LY, Zhang YQ and Wu JF: The roles of microRNA families in hepatic fibrosis. Cell Biosci 7: 34, 2017.

11. Chen Z, Zhang J,Zhang Z, Feng Z, Wei J, Lu J,Fang Y, Liang Y, Cen $\mathrm{J}$, Pan Y, et al: The putative tumor suppressor microRNA-30a-5p modulates clear cell renal cell carcinoma aggressiveness through repression of ZEB2. Cell Death Dis 8: e2859, 2017.

12. Xu B, Gong X, Zi L, Li G, Dong S, Chen X and Li Y: Silencing of DLEU2 suppresses pancreatic cancer cell proliferation and invasion by upregulating microRNA-455. Cancer Sci 110 $1676-1685,2019$.

13. Boue S, Letunic I and Bork P: Alternative splicing and evolution BioEssays 25: 1031-1034, 2003.

14. Modrek B and Lee C: A genomic view of alternative splicing. Nat Genet 30: 13-19, 2002.

15. Pan Q, Shai O, Lee LJ, Frey BJ and Blencowe BJ: Deep surveying of alternative splicing complexity in the human transcriptome by high-throughput sequencing. Nat Genet 40: 1413-1415, 2008.

16. Soergel DAW, Lareau LF and Brenner SE: Regulation of gene expression by coupling of alternative splicing and NMD. In: Madame Curie Bioscience Database. Landes Bioscience, Austin, TX, 2000-2013.

17. Edwalds-Gilbert G: Regulation of mRNA Splicing by Signal Transduction. Nat Educ 3: 43, 2010.

18. Tress ML, Abascal F and Valencia A: Alternative splicing may not be the key to proteome complexity. Trends Biochem Sci 42: 98-110, 2017.

19. Ankö ML and Neugebauer KM: Long noncoding RNAs add another layer to pre-mRNA splicing regulation. Mol Cell 39: $833-834,2010$
20. Romero-Barrios N, Legascue MF, Benhamed M, Ariel F and Crespi M: Splicing regulation by long noncoding RNAs. Nucleic Acids Res 46: 2169-2184, 2018.

21. Gil N and Ulitsky I: Production of spliced long noncoding RNAs specifies regions with increased enhancer activity. Cell Syst 7: 537-547.e3, 2018.

22. Soreq L, Guffanti A, Salomonis N, Simchovitz A, Israe Z, Bergman $\mathrm{H}$ and Soreq $\mathrm{H}$ : Long non-coding RNA and alternative splicing modulations in Parkinson's leukocytes identified by RNA sequencing. PLOS Comput Biol 10: e1003517, 2014.

23. Kiegle EA, Garden A, Lacchini E and Kater MM: A genomic view of alternative splicing of long non-coding RNAs during rice seed development reveals extensive splicing and lncRNA gene families. Front Plant Sci 9: 115, 2018.

24. Yates AD, Achuthan P,Akanni W,Allen J,Allen J,Alvarez-Jarreta J, Amode MR, Armean IM, Azov AG, Bennett R, et al: Ensembl 2020. Nucleic Acids Res 48: D682-D688, 2020

25. Williams M, Cheng YY, Kirschner MB, Sarun KH, Schelch K, Winata P, McCaughan B, Kao S, Van Zandwijk N and Reid G: Transcriptional suppression of the miR-15/16 family by c-Myc in malignant pleural mesothelioma. Oncotarget 10: 4125-4138, 2019.

26. Ma W, Zhang CQ, Dang CX, Cai HY, Li HL, Miao GY, Wang JK and Zhang LJ: Upregulated long-non-coding RNA DLEU2 exon 9 expression was an independent indicator of unfavorable overall survival in patients with esophageal adenocarcinoma. Biomed Pharmacother 113: 108655, 2019.

27. Morenos L, Chatterton Z, Ng JL, Halemba MS, Parkinso-Bates M, Mechinaud F, Elwood N, Saffery R and Wong NC: Hypermethylation and down-regulation of DLEU2 in paediatric acute myeloid leukaemia independent of embedded tumour suppressor miR-15a/16-1. Mol Cancer 13: 123, 2014.

28. Park $\mathrm{MH}$, Gutiérrez-García $\mathrm{AK}$ and Choudhury $\mathrm{M}$ : Mono-(2-ethylhexyl) phthalate aggravates inflammatory response via sirtuin regulation and inflammasome activation in RAW 264.7 cells. Chem Res Toxicol 32: 935-942, 2019.

29. Guo Y, Bai M, Lin L, Huang J, An Y, Liang L, Liu Y and Huang W: LncRNA DLEU2 aggravates the progression of hepatocellular carcinoma through binding to EZH2. Biomed Pharmacother 118: 109272,2019

30. Wu W, Zhao Y, Gao E, Li Y, Guo X, Zhao T, He W and Zhang H: LncRNA DLEU2 accelerates the tumorigenesis and invasion of non-small cell lung cancer by sponging miR-30a-5p. J Cell Mol Med 24: 441-450, 2020.

31. Zhou Y, Shi H, Du Y, Zhao G, Wang X, Li Q, Liu J, Ye L, Shen Z, Guo Y, et al: IncRNA DLEU2 modulates cell proliferation and invasion of non-small cell lung cancer by regulating miR-30c-5p/SOX9 axis. Aging (Albany NY) 11: 7386-7401, 2019.

32. Lu T, Wang R, Cai $\mathrm{H}$ and Cui Y: Long non-coding RNA DLEU2 promotes the progression of esophageal cancer through miR-30e-5p/E2F7 axis. Biomed Pharmacother 123: 109650 , 2020.

33. Zheng ZM and Wang X: Regulation of cellular miRNA expression by human papillomaviruses. Biochim Biophys Acta 1809: 668-677, 2011.

34. Corcoran MM, Hammarsund M, Zhu C, Lerner M, Kapanadze B, Wilson B, Larsson C, Forsberg L, Ibbotson RE, Einhorn S, et al: DLEU2 encodes an antisense RNA for the putative bicistronic RFP2/LEU5 gene in humans and mouse. Genes Chromosomes Cancer 40: 285-297, 2004. 